\title{
Comment justifie-t-on une croyance religieuse ? Retour sur le débat épistémologique entre William James et William Clifford
} Daniel Frey

\section{Abstract}

By looking again at the epistemological debate between James and Clifford, which James seemed to win, this study aims to show that an epistemology of a properly understood religious belief, that is : one which seeks neither to justify religious beliefs rationally nor to dismiss them out of hand, can only proceed by describing the intertwining of the reasons for belief and the will to believe through which human subjects legitimise all their beliefs.

\section{Résumé}

En revenant sur le débat épistémologique entre James et Clifford, dont l'issue semble favorable au premier, la présente étude entend montrer qu'une épistémologie de la croyance religieuse bien comprise, c'est-à-dire qui ne cherche ni à justifier rationnellement les croyances religieuses ni à les condamner, ne peut que tenter de décrire l'entrelacement des raisons de croire et de la volonté de croire par lesquels les sujets légitiment leurs croyances.

\section{Citer ce document / Cite this document :}

Frey Daniel. Comment justifie-t-on une croyance religieuse ? Retour sur le débat épistémologique entre William James et William Clifford. In: Revue d'histoire et de philosophie religieuses, 96e année n4, Octobre-Décembre 2016. pp. 381-401; doi : https://doi.org/10.3406/rhpr.2016.2013

https://www.persee.fr/doc/rhpr_0035-2403_2016_num_96_4_2013

Fichier pdf généré le 09/12/2019 


\title{
COMMENT JUSTIFIE-T-ON UNE CROYANCE RELIGIEUSE ? Retour sur le débat épistémologique
entre William James et William Clifford*
}

\author{
Daniel Frey
}

Faculté de Théologie protestante (EA 4378) - Université de Strasbourg

9 place de l'Université - F-67084 Strasbourg Cedex

\begin{abstract}
Résumé : En revenant sur le débat épistémologique entre James et Clifford, dont l'issue semble favorable au premier, la présente étude entend montrer qu'une épistémologie de la croyance religieuse bien comprise, c'est-à-dire qui ne cherche ni à justifier rationnellement les croyances religieuses ni à les condamner, ne peut que tenter de décrire l'entrelacement des raisons de croire et de la volonté de croire par lesquels les sujets légitiment leurs croyances.
\end{abstract}

Abstract: By looking again at the epistemological debate between James and Clifford, which James seemed to win, this study aims to show that an epistemology of a properly understood religious belief, that is : one which seeks neither to justify religious beliefs rationally nor to dismiss them out of hand, can only proceed by describing the intertwining of the reasons for belief and the will to believe through which human subjects legitimise all their beliefs.

\section{DEUX REGARDS SUR LA CROYANCE}

Les 'faits' sont les limites de la connaissance humaine, limites établies en vue de cette connaissance et non par elle.

William James ${ }^{1}$.

La réalité ne sort pas de nos crânes telle Athéna, sortie toute armée du crâne de Jupiter. C'est notre intelligence qui tranche dans l'infinité des variations des phénomènes, c'est notre regard qui compose les faits en données intelligibles, tout comme il établit les constellations dans la nuit étoilée. Les étoiles, après tout, ne forment des figures célestes que pour les observateurs humains sur notre terre : à l'autre bout de la galaxie, elles en forment d'autres.

\footnotetext{
* Cette étude reprend et développe la Leçon de rentrée donnée le 27 septembre 2016 à la Faculté de Théologie protestante de l'Université de Strasbourg.

${ }^{1}$ James, 2005 [1897], p. 264.
} 
Il en va de même, mutatis mutandis, pour les objets que nous constituons en savoirs objectifs : tout savoir sur l'objet dépend à la fois d'un positionnement, d'une observation à partir de ce positionnement et de l'outillage employé. C'est devenu évident lorsque l'examen porte sur un fait relevant des sciences humaines, c'est-àdire un fait composé d'éléments de natures diverses (linguistique, historique, sociologique, psychologique), mais cela n'est pas moins vrai pour un fait physique, car la connaissance du fait physique suppose toujours que l'on ne prenne pas en compte toutes les données, afin de sélectionner celles qui seront pertinentes pour l'étude. La connaissance d'un objet va donc de pair avec la sélection des traits qui permettent un traitement le plus objectif possible de cet objet. La connaissance est une forme d'abstraction, de sorte que James est fondé à écrire : « Pour apercevoir l'univers sous [un certain] degré de rationalité, il nous a fallu éliminer déjà une grande part de son contenu ${ }^{2} »$.

Il n'en va pas autrement du fait que constitue la «croyance religieuse ». Nous aimerions montrer ici comment le jugement philosophique porté sur la croyance religieuse peut varier en fonction de l'épistémologie de la croyance mise en œuvre, c'est-à-dire en fonction des critères épistémiques auxquels le philosophe soumet la croyance religieuse dans le but de déterminer quelle forme de connaissance elle permet. En effet, s'il attend de la croyance qu'elle autorise une connaissance vérifiable, l'épistémologue sera enclin, comme William Clifford (1845-1879) dans L'éthique de la croyance ${ }^{3}$, à dénier à la croyance toute valeur épistémique. Si au contraire il soutient, comme William James (1842-1910) dans La volonté de croire $^{4}$ - ouvrage précisément destiné à répondre à Clifford dixhuit années après sa mort -, que l'attente de preuves suffisantes est indue parce qu'elle est impossible à satisfaire en raison même de la nature de la croyance, il tendra à justifier la croyance comme un type de connaissance imparfait, certes, mais inévitable. Selon James, l'in-croyance (dire « je ne crois pas que... ») revient, dans les faits, à se déterminer face à un objet de croyance, c'est-à-dire à opposer une certitude indémontrable à une autre. La croyance et l'incroyance ont donc un véritable air de famille et s'opposent moins entre elles qu'elles ne s'opposent au doute et à l'enquête, lesquelles suspendent le jugement sine $d i e^{5}$. Mais ce doute et cette enquête restent

\footnotetext{
${ }^{2}$ James, 2005 [1897], p. 260.

3 «The Ethics of Belief» (Clifford, 1879 [1877]).

${ }^{4}$ La volonté de croire est un ensemble d'essais touchant, pour les quatre premiers, à la question de la croyance religieuse, paru en 1897 sous le titre The Will to Believe and Other Essays in Popular Philosophy. L'auteur avait initialement prévu de lui donner pour titre Right to believe, c'est-à-dire Le droit de croire (cf. Meulders, 2010, p. 227).

${ }^{5}$ Voir James, 2010 [1889], p. 102-103.
} 
eux-mêmes rarement ouverts : tout l'enjeu de la lecture que James fait de Clifford est justement de montrer que ce dernier n'est pas durablement sceptique et qu'il a choisi de croire que la foi chrétienne est vide. S'il en est ainsi, nous le verrons, c'est parce que la volonté de se déterminer - à croire ou à ne pas croire - est liée à notre "nature passionnelle 6 ".

Nous choisissons dans cette étude de reprendre et de commenter le débat fameux entre Clifford et James (sans nous engager dans son détail), parce qu'il renvoie à deux regards très différents portés sur la croyance et nous semble de nature à éclairer le mode de savoir très spécifique qu'est la croyance religieuse. Dans la perspective que nous adoptons ici, l'épistémologie de la croyance religieuse est donc bien dévolue à "l'examen des raisons de croire », cher à Roger Pouivet ${ }^{7}$, mais nous n'entreprendrons pas cet examen de la façon dont l'entend cet auteur. En effet, prendre pour objet les raisons de croire n'oblige pas, selon nous, à procéder à une évaluation philosophique des «prétentions à la rationalité ${ }^{8}$ » de la croyance religieuse. Dans cette évaluation, l'épistémologue cherche à valider sur le plan épistémique les raisons de croire alléguées par les croyants, dans le but de justifier la croyance. Il s'agit bien plutôt, dans une perspective à la fois plus large et plus distanciée, de décrire diverses entreprises philosophiques permettant de penser la croyance religieuse en tant que mode de connaissance spécifique, à partir d'épistémologies aussi dissemblables que celles de Clifford, de James, ou de Wittgenstein, pour n'indiquer que les auteurs dont il sera question ici.

\section{CROIRE, C'EST « TENIR POUR VRAI »}

La croyance a toujours une portée cognitive. Elle est une affirmation de vérité à l'appui de laquelle il est possible de donner des raisons (raisons de croire), qu'il s'agisse de la croyance au sens général et profane du terme (croire en l'issue favorable d'un conflit, par exemple) ou d'une croyance religieuse (par exemple la croyance en l'existence d'un Être divin auquel toutes choses se rapporteraient). En effet, le terme de " croyance » est tout autant légitime pour désigner une croyance profane qu'une croyance spécifiquement

\footnotetext{
${ }^{6}$ James, 2005 [1897], p. 47 (c'est James qui souligne). Rousseau l'écrivait déjà dans Émile : « le doute sur les choses qu'il nous importe de connaître est un état trop violent pour l'esprit humain ; il n'y résiste pas longtemps, il se décide malgré lui d'une manière ou d'une autre, et il aime mieux se tromper que ne rien croire » (Rousseau, 1969 [1762], p. 568 - nous modernisons l'orthographe).

${ }^{7}$ Pouivet, 2002, p. 29.

${ }^{8}$ Pouivet, 2002, p. 12, cf. Pouivet, 2003.
} 
religieuse, comme l'atteste son étymologie : croyance vient de créance, provenant du latin credentia, issu de credere, verbe d'origine indoeuropéenne relevant de la langue religieuse qui, dès l'origine, a connu des emplois profanes (mettre sa confiance en quelqu'un/en quelque chose ; croire quelqu'un / croire quelque chose) ${ }^{9}$.

On le sait, la croyance a souvent eu mauvaise réputation en philosophie, parce que le degré de savoir qu'elle contient semble faible, par comparaison à la certitude de type mathématique ; d'autant plus faible, précisément, que le degré de certitude afférent au sujet est fort. Cependant, même Platon, que l'on invoque généralement dès qu'il s'agit de critiquer la doxa - l'opinion, la croyance au sens profane du terme - n'a pu manquer de reconnaître, dans son dialogue intitulé Théétète, que toute croyance n'est pas condamnable, et surtout que la croyance en tant que telle n'est pas évitable. Bien sûr, chez Platon le modèle épistémologique est celui des mathématiques, et plus précisément de la géométrie, qui traite de réalités nécessaires, inconditionnelles et immuables. Mesurée à cette aune, toute opinion, toute croyance est évidemment en défaut. Mais il s'agit précisément d'un idéal que seule la géométrie peut atteindre dans notre condition humaine : si l'opinion/croyance/doxa est inférieure à la science ainsi comprise, elle n'est en revanche pas synonyme d'ignorance, et c'est là, précisément, que commence tout le problème. On voit très bien, à la lecture du Théétète, que Platon (par l'intermédiaire de son porte-parole, Socrate) est conscient qu'il est inévitable que nous, sujets humains, nous prétendions toujours dire vrai, même là où la vérité nous échappe : " nous sommes bien forcés de faire ce que notre nature nous commande et de dire toujours ce qui nous paraît vrai ${ }^{10}$. »

Essayons d'énoncer la même idée, sans Platon cette fois. En toute croyance un jugement de vérité est exprimé : dire "je crois que $p »$ (selon la formule classique des épistémologues ${ }^{11}$ ), c'est dire que " $p$ » est vrai, qu'il existe, ou le pourrait. L'expérience la plus ordinaire de la croyance est bien celle d'un jugement de vérité qui peut être justifié. C'est ainsi que nous disons « je crois qu'il va pleuvoir » en observant le ciel un soir d'été, parce que nous avons souvent vu un tel ciel engendrer de la pluie, même si en d'autres occasions les signes ont aussi pu s'avérer trompeurs. Nous disons également, en attendant un ami devant en aider un autre pour son déménagement, "je crois qu'il viendra», parce que la connaissance que nous avons de cet ami nous amène à penser qu'il est

\footnotetext{
${ }^{9}$ Voir Rey, 1998, p. 956-957.

${ }^{10}$ Théétète $171 \mathrm{c}$, voir Platon, 1967, p. 106.

${ }^{11}$ Un seul exemple : Engel, 1995, p. 26, passim.
} 
généralement fidèle à ses engagements et qu'il aura a priori, en cas de difficultés dont on ignore tout, chercher à l'être. Bien sûr, la croyance n'exprime pas ici un savoir indubitable - car qui peut prédire le temps, les circonstances ou le comportement des personnes ? -, mais une probabilité forte, en l'absence de tout autre moyen de vérification. La croyance ordinaire, prise ici dans son sens le plus faible, exprime par nature une forme de connaissance d'un type particulier. Nous disons « je crois... » pour exprimer un savoir incertain... mais c'est un certain savoir quand même! Selon les règles de nos échanges linguistiques, il ne serait en effet guère acceptable de dire « je crois qu'il viendra mais je ne crois pas qu'il viendra » (ou je crois $p$ mais je crois $\neg p$.), précisément parce qu'il est attendu qu'en disant cela, l'on exprime une connaissance qui a ses raisons et peut donc se justifier. Dans le même temps, il est admis que la croyance exprime un pari sur une probabilité plutôt qu'une science indubitable, sans quoi il faut dire, à parler strictement, non pas « je crois », mais « je sais ».

En théorie, une chose est le degré de probabilité du savoir relatif à un objet, sur lequel porte l'énoncé de croyance, une autre le degré de certitude du sujet attestant un tel savoir ${ }^{12}$. Mais les deux échelles se recoupent le plus souvent, ce qui fait que l'on peut être profondément convaincu d'une réalité indémontrable aux yeux d'autrui (par exemple le caractère prémonitoire d'un rêve que l'on a fait) ou faiblement convaincu de l'existence d'une réalité ailleurs parfaitement vérifiable. Ainsi, je crois qu'il pourrait éventuellement rester des places pour un concert assez proche auquel je compte assister. Je le crois en tout cas possible jusqu'à ce que je puisse le vérifier à la billetterie. Il n'y aura alors plus à croire, mais à se réjouir de cette bonne fortune, ou non.

On se doute que si la croyance religieuse est comme toute croyance une affirmation de vérité, elle porte en revanche sur des objets dont l'existence ne peut être vérifiée sur le mode ordinaire de la vérification empirique et/ou scientifique, en raison du caractère (par définition) suprasensible du divin. Même religieusement parlant, on ne croit qu'en l'absence de tout savoir indubitable. Je ne peux affirmer l'existence de l'objet de ma croyance religieuse que pour autant que cet objet n'est pas susceptible d'être connu objectivement, conformément aux critères intersubjectifs de cette connaissance objectivement satisfaisante que l'on appelle proprement le « savoir ». A contrario, je n'ai pas besoin de croire que l'eau bout

\footnotetext{
${ }^{12}$ Voir sur ce point le magistral article «Croyance » que Paul Ricœur a rédigé pour 1'Encyclopaedia Universalis : Ricœur, 2000 [1969], p. 311.
} 
à 100 degrés ; je l'ai appris et expérimenté en classe à l'aide d'un réchaud, d'une casserole d'eau et d'un thermomètre.

Ce disant, nous reprenons la lumineuse distinction opérée par Kant dans la Critique de la raison pure entre trois types possibles de croyances, ou plutôt, pour reprendre la traduction ingénieuse de Delamarre et Marty, de " créances » (Fürhwahrhalten) ${ }^{13}$ : la simple opinion (Meinen) marque une affirmation toute personnelle, clairement insuffisante, même aux yeux du sujet lui-même (ainsi je crois que l'hiver sera neigeux). La foi (Glaube) désigne la croyance religieuse à strictement parler, qui se sait objectivement insuffisante sur le plan de la communication entre les sujets de raison que nous sommes, mais suffisante sur le plan subjectif (je crois au Karma; je crois à la Résurrection) ${ }^{14}$. Alors que le savoir (Wissen, qui est aussi, notons-le, une forme de créance) est suffisant aussi bien objectivement que subjectivement. Cette série de distinction peut être représentée schématiquement comme suit :

\begin{tabular}{|c|c|c|c|c|}
\hline $\begin{array}{c}\text { Les trois types } \\
\text { de créances } \\
\begin{array}{c}\text { Fürhwahrhalten) } \\
\text { selon Kant }\end{array}\end{array}$ & $\begin{array}{c}\text { Créance } \\
\text { insuffisante } \\
\text { objectivement }\end{array}$ & $\begin{array}{c}\text { Créance } \\
\text { insuffisante } \\
\text { subjectivement }\end{array}$ & $\begin{array}{c}\text { Créance } \\
\text { suffisante } \\
\text { subjectivement } \\
\text { = Conviction } \\
\text { (pour moi- } \\
\text { même) }\end{array}$ & $\begin{array}{c}\text { Créance } \\
\text { suffisante } \\
\text { objectivement } \\
\text { = Certitude } \\
\text { (pour chacun) }\end{array}$ \\
\hline Opinion & $\mathrm{x}$ & $\mathrm{x}$ & - & - \\
\hline Foi & $\mathrm{x}$ & - & $\mathrm{x}$ & - \\
\hline Savoir & - & - & $\mathrm{x}$ & $\mathrm{x}$ \\
\hline
\end{tabular}

Il n'y a donc pas de place pour la foi là où l'on prétend connaître objectivement, et c'est le sens de la parole si profonde de Kant, dans la seconde préface à la Critique de la raison pure, où il commente sa propre destruction des preuves ontologiques de l'existence de Dieu: «je devais donc supprimer le savoir, pour trouver une place pour la $f o i^{15} »$. Cela signifie-t-il que dès qu'il s'agit de croyance, l'arbitraire le plus éhonté a droit de cité ? Certainement pas. Les raisons de croire existent en toute croyance, et

\footnotetext{
${ }^{13}$ Voir Kant, 1980 [1781-A820/1787-B848], p. 1376-1378; on trouve dans la Critique de la faculté de juger (\$91) un propos comparable et complémentaire, cf. Kant, 1968 [1790], p. 271. Delamarre et Marty, traducteurs de la Critique de la raison pure dans l'édition de la Pléiade, choisissent en effet de traduire Fürhwahrhalten par «créance » afin de ne pas induire de confusion entre le genre et l'espèce (ibid., p. 1735).

${ }^{14} \mathrm{~S}$ 'il est fréquent, notamment depuis la théologie de Karl Barth, d'opposer la foi à la croyance - la première étant spécifique à la révélation biblique, la seconde relevant de la religion conçue comme effort humain pour s'élever jusqu'au divin -, nous choisissons dans la présente étude, à la suite de Kant, de l'inclure dans le phénomène de la croyance, qui jouit d'un statut générique.

${ }^{15}$ Kant, 1980 [1787-BXXX], p. 748 (c'est Kant qui souligne).
} 
c'est précisément ce qui différencie ce phénomène de l'aimable affabulation et du délire pathologique, de sorte que c'est de toute croyance qu'il faudrait dire, avec l'Apôtre, qu'elle doit s'apprêter à rendre raison $(1 P 3,15)$ à ceux qui lui demandent de se justifier. En se montrant soucieux de distinguer ce qui fonde le caractère subjectivement suffisant de la croyance religieuse aux yeux du croyant de ce qui rend le savoir objectivement et subjectivement suffisant aux yeux de tous, Kant nous met sur la voie d'une épistémologie de la croyance qui tient compte des raisons de croire qui ne sont pas strictement rationnelles. Contrairement à ce qu'affirment les épistémologues issus du courant de la philosophie analytique de la religion, l'épistémologie de la croyance ne doit pas être dévolue au seul examen des causes rationnelles de la croyance religieuse. Deux arguments peuvent ici être avancés en faveur de cette thèse.

Le premier argument est que l'examen des raisons doit résider tout autant - voire davantage - dans l'attention portée à tout ce qui nous semble valoir comme raisons, tout simplement parce qu' aucun croyant, dans les faits, ne limite ses raisons de croire aux raisons rationnelles et raisonnantes, mais qu'il y inclut de raisons de tous ordres, qui fonctionnent comme des motifs de crédibilité et non comme des preuves. Font partie intégrante de ces raisons non contraignantes, de ces gages de crédibilité, certaines images du monde héritées des traditions sociales, culturelles et religieuses. Il s'agit là, bien sûr, des justifications spécifiquement religieuses, issues de la tradition de foi à laquelle le sujet se reconnaît appartenir et se référant le plus souvent à des arguments tirés de ses livres saints et des pratiques religieuses transmises de génération en génération. Il n'y a aucune raison de soustraire ces raisons à l'examen, car ce sont celles qui emportent l'adhésion du croyant et se transmettent au sein de la communauté de croyance à la faveur d'une redécouverte perpétuelle.

Le second argument pour ne pas limiter l'épistémologie de la croyance religieuse au seul examen de la rationalité du croire tient précisément au fait que, lorsqu'elle est entreprise, cette épistémologie met en œuvre un traitement des raisons de croire qui déroge au canon de pure rationalité. C'est encore le cas, tout récemment, de l'épistémologie de la croyance religieuse mise en œuvre par Roger Pouivet, qui a procédé à un examen systématique de la " légitimité épistémologique ${ }^{16}$ " de la croyance chrétienne en Dieu, d'où il ressort, selon lui, que les raisons de croire des chrétiens n'ont finalement pas à être évaluées en fonction des «normes $a$

\footnotetext{
${ }^{16}$ Pouivet, 2013, p. 9.
} 
priori de légitimité épistémologique ${ }^{17} \gg$ (celles de Descartes, Locke, Kant), mais bien plutôt en référence aux « vertus intellectuelles ou épistémiques ${ }^{18} \gg$. De ce point de vue, que Pouivet oppose à l'éthique de la croyance de Clifford, elles seraient épistémologiquement fondées. En dépit de ses indéniables qualités descriptives, qu'il nous est malheureusement impossible de détailler, cette approche épistémologique, fondée sur un tel traitement d'exception, souffre d'un aspect tout aussi militant que celle de Clifford, dans le sens inverse d'une justification théiste de la croyance chrétienne.

Que les raisons rationnelles de croire ne suffisent jamais, mais appellent - même là où le penseur croyant veut et croit entreprendre une démonstration purement rationnelle - un traitement extraordinaire, c'est ce qui apparaît déjà dans la fameuse démonstration ontologique de l'existence de Dieu entreprise par Anselme de Cantorbéry au $\mathrm{XI}^{\mathrm{e}}$ siècle. Dans son célèbre Proslogion, Anselme entendait démontrer l'existence de Dieu à «l'Insensé » (disant en son cœur, selon le Psaume 14, «Il n'y a pas de Dieu ») ${ }^{19}$ par le fait que si l'on définit Dieu comme étant l'Être tel que rien de plus grand ne peut exister, il existe nécessairement, car s'il lui manquait l'existence, il ne serait pas conforme à sa définition. Dans Pour la défense de l'insensé, Gaunilon, contemporain d'Anselme, le prend au mot : puisqu'Anselme est parti du principe qu'il faut convaincre un «Insensé » fictif de façon purement logique ${ }^{20}$, le lecteur peut à bon droit estimer - à l'instar de Gaunilon - qu'Anselme « ne tient pas suffisamment compte de celui à qui il parle ${ }^{21} »$ lorsqu'il s'adresse à un tel contradicteur imaginaire :

Pour moi en effet, je ne dis pas encore - bien plus même, je nie ou au moins je doute qu'Il soit plus grand que n'importe quel objet existant vraiment; et je ne Lui concède pas d'autre existence - si l'on peut parler d'existence - que celle d'un objet encore inconnu, que l'esprit s'efforce de se représenter à l'audition d'un simple mot. Comment donc me prouvera-t-on de cette manière que ce plus grand existe en réalité parce qu'il est évident qu'Il est plus grand que tous, alors que, jusqu'ici, je Le nie - ou du moins en doute -, si bien que je ne puis dire que ce plus grand soit dans mon intelligence ou ma pensée au même titre où y sont beaucoup de choses fausses ou douteuses? Il faut, en effet, que l'on me convainque d'abord que ce 'plus grand' existe quelque part en

${ }^{17}$ Pouivet, 2013, p. 18.

18 Pouivet, 2013, p. 19.

${ }^{19}$ Anselme, 1947 [XI ${ }^{\mathrm{e}}$ siècle], p. 179.

${ }^{20}$ Même s'il est évident, ne serait-ce qu'en vertu de l'adresse à Dieu ouvrant le Proslogion, que la démonstration d'Anselme relève encore et fondamentalement de la théologie chrétienne.

${ }^{21}$ Gaunilon, 1947 [XI ${ }^{\mathrm{e}}$ siècle], p. 204. 
réalité - alors seulement de ce qu'Il est plus grand que tout, il sera certain qu'Il existe aussi réellement ${ }^{22}$.

C'est précisément ce qu'affirmera Kant huit siècles plus tard. L'argumentation d'Anselme (et, mutatis mutandis, celle de Descartes à sa suite) consiste en effet à soutenir que l'Etre nécessaire est " quelque chose dont la non-existence est impossible ${ }^{23}$ ». La réponse kantienne vise d'abord à dire qu'il est possible de ne pas penser $d u$ tout un Être nécessaire, comme il est possible de ne pas penser que la somme des angles d'un triangle équivaut à 180 degrés en ne pensant pas de triangle du tout ${ }^{24}$. Ensuite et surtout, l'argument ontologique signifie que l'Être parfait existe parce que l'existence est une perfection, ou parce qu'on ne saurait concevoir un Être parfait qui n'existe pas. À cela Kant répond que l'existence ne peut être considérée comme une perfection pensée, telle que la toutepuissance par exemple, mais seulement comme une position qui ne peut se vérifier qu'empiriquement. L'objet et sa position ne contiennent rien de plus que son concept : « cent thalers effectifs ne contiennent rien de plus que cent thalers possibles ${ }^{25} »$. Or, comme l'affirme le philosophe de Königsberg, si « je pense un être comme la suprême réalité (sans défaut), il reste toujours à savoir si cet être existe ou non. En effet, bien qu'à mon concept il ne manque rien du contenu réel possible d'une chose en général, il manque cependant encore quelque chose au rapport à tout mon état de pensée, à savoir que la connaissance de cet objet soit aussi possible a posteriori ${ }^{26} \gg$. Il est donc impossible de prouver, hors du champ de l'expérience, $a$ priori, l'existence de Dieu, comme il est impossible, pour la même raison, de prouver son inexistence ${ }^{27}$.

Ce bref passage par les critiques de Gaunilon et de Kant montre que l'entreprise de justification rationnelle d'une croyance (ici, la croyance en l'existence de Dieu) repose paradoxalement - et de façon plus ou moins consciente - sur un traitement extraordinaire des arguments, preuve, à nos yeux, que les raisons religieuses de croire s'insinuent (et comment ne le ferait-elle pas ?) dans les raisons censées être objectivement satisfaisantes. Le paradoxe de la théologie naturelle en général et des preuves de l'existence de Dieu en particulier - paradoxe qui se retrouve dans l'épistémologie religieuse

\footnotetext{
${ }^{22}$ Gaunilon, 1947 [XI ${ }^{\mathrm{e}}$ siècle], p. 205. On voit qu'ici Gaunilon assume et retourne contre elle la position de l'Insensé (ou plutôt de l'agnostique) que la rhétorique anselmienne a elle-même créée. Il va de soi que cette figure agnostique est fictive, et d'ailleurs historiquement et socialement bien improbable en plein Moyen-Âge.

${ }^{23}$ Kant, 1980 [1781-A592/1787-B620], p. 1210.

${ }^{24}$ Cf. Kant, 1980 [1781-A593/1787-B621], p. 1211.

${ }^{25}$ Kant, 1980 [1781-A599/1787-B627], p. 1215.

${ }^{26} \mathrm{Kant}, 1980$ [1781-A600/1787-B628], p. 1216.

${ }^{27}$ Kant, 1980 [1781-A641/1787-B669], p. 1246.
} 
de la croyance religieuse -, c'est qu'elles n'entendent reposer que sur la raison alors qu'elles prennent pour objet un Être qui par définition se situe en dehors de l'usage que nous pouvons faire de la raison. En matière de croyance religieuse, ce qui nous convainc rationnellement (de jure), c'est-à-dire ce qui est censé justifier nos croyances aux yeux de nos interlocuteurs comme aux nôtres, dépend ainsi dans une large mesure de l'attitude que - de facto - nous avons déjà adoptée à l'égard de la croyance en question.

S'il en est ainsi, c'est que le sujet de la croyance ne peut empêcher que ce en quoi il croit déjà détermine le choix et la force des arguments rationnels qu'il entend avancer à l'appui de sa croyance. De la même manière, ce n'est pas faire injure à la mémoire de Clifford que de préciser qu'il était lui-même un libre penseur actif, organisant par exemple un congrès de libre pensée en 1878, à une date coïncidant quasiment avec l'anniversaire de la mort de Voltaire ${ }^{28}$. Pour un rationaliste comme Clifford, on ne peut croire que si les raisons que l'on allègue peuvent être abandonnées à l'occasion d'un examen critique : or cette épistémologie de la croyance présuppose que la réalité des objets de croyance ne peut $q u$ 'être prise en défaut par l'exercice d'un jugement sain! James est, selon nous, convainquant lorsqu'il avance que l'épistémologie de la croyance de Clifford est elle-même la preuve que la relation à la vérité met en jeu notre "nature passionnelle 29 ", et que toute relation à la croyance, même épistémologique, est déjà une prise de position émotionnelle.

\section{LA NATURE PASSIONNELLE DE LA CROYANCE}

\section{William Clifford: The Ethics of Belief}

The Ethics of Belief, paru en 1877, est le texte le plus connu du philosophe et mathématicien britannique William K. Clifford. Ce ne sont pas seulement des considérations épistémologiques (pour croire il faut des preuves) qui enjoignent Clifford à faire preuve de scepticisme à l'endroit des considérations religieuses, mais, comme l'indique fort bien le titre, des considérations morales. Clifford prend l'exemple d'un armateur qui, bien qu'il soit conscient de l'état délabré de son navire, repousse ses doutes et veut croire qu'il traversera cette fois encore l'océan, comme il l'a déjà fait par le passé, et parviendra sans encombre au port ${ }^{30}$. Le bateau sombre

\footnotetext{
${ }^{28}$ C'est Jacques Bouveresse qui atteste cela : voir Bouveresse, 2015, p. 5.

${ }^{29}$ James, 2005 [1897], p. 47 (c'est James qui souligne).

${ }^{30}$ Cf. Clifford, 1879 [1877], p. 177-178.
} 
finalement, ses occupants sont noyés : cette croyance était donc non seulement indue, mais immorale. La croyance de l'armateur constituait une mise en berne éhontée des principes de moralité et de prudence; elle l'arrangeait bien et n'a fait que l'autoriser à se soustraire à son devoir d'examiner attentivement l'état du navire et de mettre en œuvre des réparations qui s'imposaient.

L'éthique de la croyance de Clifford semble bien interdire la croyance religieuse, puisque les preuves suffisantes requises par son principe y feront toujours défaut. C'est aussi l'avis de Jacques Bouveresse, pour qui Clifford « est convaincu qu'elle [la croyance religieuse] ne résisterait pas à une application rigoureuse et conséquente du principe » qu'il formalise ${ }^{31}$. Dans le même temps, Clifford indique qu'une position systématiquement sceptique n'est guère tenable, et que nous devons soutenir un certain nombre de croyances, par exemple celle voulant qu'il est mauvais de mentir même si nous n'avons jamais assez d'expérience, dans notre vie personnelle, pour justifier cette croyance ${ }^{32}$. Comme le note encore Jacques Bouveresse, Clifford s'inscrit clairement dans la tradition de l'Aufklärung exigeant de toute tradition qu'elle accepte d'être interrogée, mise en doute, car elle-même est destinée à nous accompagner dans notre effort pour être humains, et non destinée à être répétée sans examen ${ }^{33}$. C'est finalement, aux yeux de Clifford, une offense envers l'humanité elle-même que de se refuser à examiner une croyance religieuse au motif qu'elle a été reçue via l'autorité de la tradition et qu'elle pourrait bien ne pas sortir vivante de l'examen.

On lit en effet dans The Ethics of Belief ce principe discuté dans de nombreux essais d'épistémologie de la croyance :

Pour résumer : il est toujours mauvais, partout et pour n'importe qui, de croire quoi que ce soit sur la base d'une preuve insuffisante. Qu'un homme, tenant à une croyance qu'on lui a apprise dans son enfance, rabaisse et repousse tout doute s'élevant dans son esprit, évite sciemment la lecture de livres et la compagnie d'hommes qui la mettraient en question et la discuteraient, et regarde comme impies les questions auxquelles il n'est pas aisé de répondre sans la perturber : la vie de cet homme constituerait tout du long un péché contre l'humanité ${ }^{34}$.

\footnotetext{
${ }^{31}$ Bouveresse, 2015, p. 5.

${ }^{32}$ Cf. ibid., p. 188. Non sans à-propos, Pascal Engel parle à son endroit de «puritanisme scientifique » (Engel, 2007, p. 135).

${ }^{33}$ Cf. Bouveresse, 2015, p. 20.

34 «To sum up : it is wrong always, everywhere, and for anyone, to believe anything upon insufficient evidence. If a man, holding a belief which he was taught in childhood or persuaded of afterwards, keeps down and pushes away any doubts which arise about it in his mind, purposely avoids the reading of books and the company of men that call in question or discuss it, and regards as impious those questions which cannot easily be asked without disturbing it - the life of that man is one long sin against mankind » (Clifford, 1879 [1877], p. 186-187; nous traduisons). Cette version de ce que l'on appelle couramment le « principe
} 
Toutefois, aussi fameux soit-il, l'exemple de l'armateur n'est pas tout à fait adéquat pour penser la croyance religieuse, puisque, d'une part, celle-ci ne pourra jamais donner lieu à des vérifications du même ordre, et que, d'autre part, elle n'est sans doute pas aussi consciemment choisie que ne l'a été la croyance au bon cours des choses chez cet armateur. Cet exemple et l'ensemble de l'argumentation de Clifford supposent clairement un a priori défavorable à la croyance religieuse, tandis que celle de James, qui lui répond dixhuit années après sa mort, suppose tout aussi clairement un a priori favorable. Il ne s'agit pas ici d'opposer dos-à-dos deux épistémologies de la croyance, mais de saisir laquelle est la plus conforme à la réalité de la croyance - à la croyance telle que les sujets la justifient concrètement. À ce compte-là, c'est l'épistémologie de James présentée dans La Volonté de croire (1897), dont les quatre premiers essais ont pour objet de défendre la légitimité de croyance religieuse ${ }^{35}$, qui l'emporte.

\section{William James : La volonté de croire}

Reconnaissons toutefois que l'épistémologie de James ne présente pas toute la clarté désirée. Elle est assez imprécise et répétitive, et, si elle vise le plus souvent Clifford, elle ne le fait parfois que par allusion. Aussi peut-on se proposer de suppléer à ces manques formels en synthétisant un certain nombre d'analyses sous forme de propositions, tout en renvoyant au texte de James lorsqu'il s'avère assez précis :

Nous appellerons hypothèse tout ce qui est proposé à notre croyance ; et nous distinguerons parmi les hypothèses celles qui sont en quelque sorte vivantes de celles qui sont mortes. Une hypothèse vivante est celle qui se pose comme une véritable possibilité devant l'entendement auquel elle est soumise : si je vous demande de croire au Mahdi, une telle idée $[\ldots]$ ne s'éclaire point de la lumière des choses croyables; en tant qu'hypothèse, elle est absolument morte. Pour un Arabe cependant, alors qu'il ne serait pas un sectateur du Mahdi ${ }^{36}$, 1 'hypothèse fait

de Clifford » se trouve à peu près dans le premier tiers du texte ; celle citée la plupart du temps ( «It is wrong in all cases to believe on insufficient evidence; and where it is presumption to doubt and to investigate, there it is worse than presumption to believe » [ « il est mauvais dans tous les cas de croire sur la base d'une preuve insuffisante ; et là où c'est de la présomption de douter et de chercher, c'est pire que de la présomption que de croire »], ibid., p. 211) en constitue les dernières lignes.

${ }^{35}$ Cf. James, 2005 [1897], p. 35. Il n'est pas inutile d'indiquer que l'ensemble de l'ouvrage forme aux yeux de James essai d'" empirisme radical » (James, 2005 [1897], p. 33) : empirisme signifie ici que ses conclusions sont d'emblée présentées comme des hypothèses que l'expérience future pourra démentir ; et radical indique que même le monisme y apparait comme une hypothèse. L'empirisme de James le porte au pluralisme, mais n'est pas selon lui un scepticisme : le pluralisme est simplement selon lui une hypothèse plus crédible, plus conforme à l'expérience de l'univers. Tel qu'il nous apparaît, l'unité du monde est « celle d'une collection» (James, 2005 [1897], p. 33).

${ }^{36}$ L'Envoyé d'Allah, attendu pour parachever l'œuvre du Prophète Mahomet. 
partie des possibilités de sa pensée, elle est vivante. Ceci prouve que la vitalité ou le défaut de vie d'une hypothèse n'expriment point des propriétés intrinsèques, mais un rapport entre l'hypothèse et chaque penseur individuel ; elles se mesurent à la volonté d'agir qu'elles provoquent ${ }^{37}$.

Ainsi le sujet ne se donne-t-il donc pas les croyances qu'il lui plaît, et la croyance ne se justifie pas par les qualités 'objectives' de l'objet de croyance, mais par son lien vivant avec le sujet. Lorsque ce lien n'est pas vivant, il n'y a pas, à proprement parler, de croyance pour ce sujet : l'hypothèse ne fait pas sens. Il est essentiel de saisir que, pour James, nous ne nous abstenons jamais de croire : certaines croyances sont vives à nos yeux, parce qu'elles nous portent à agir, et parce que d'autres ne le sont pas et restent « lettre morte ». Dans un essai de 1889 intitulé « La psychologie de la croyance », James notait déjà que « nous ne nions jamais une chose si ce n'est pour la raison que nous croyons en quelque chose d'autre qui la contredit ${ }^{38}$ ». Les croyances renvoient donc à des incroyances, au sein d'un système qui porte à agir : croire que... c'est aussi ne pas croire que... et agir en conséquence. Une parenté existe entre ce propos et celui de Wittgenstein, qui estimera, à la fin de sa vie, que nous héritons de nos certitudes, lesquelles ne sont pas systématiquement interrogées mais permettent de nous représenter le monde et d'y agir : « Ce à quoi je m'en tiens fermement, ce n'est pas une proposition, mais un nid de propositions ${ }^{39}$. »

Le second point mis en avant par James est que c'est clairement « notre nature passionnelle et volitive » qui se trouve « à la racine de toutes nos convictions ${ }^{40} »$, y compris celles qui s'appuient sur une volonté de ne rien recevoir sans preuve : " nous pouvons observer que nous croyons, et c'est à peine si nous savons comment et pourquoi », tant « la crainte et l'espoir, les préjugés et les passions, l'imitation et l'esprit de parti, l'influence de la caste et du

\footnotetext{
${ }^{37}$ James, 2005 [1897], p. 40 (c'est James qui souligne).

${ }^{38}$ James, 2010, [1889], p. 102. Cet essai sera en grande partie repris dans les Principles of Psychology (Principes de la psychologie) parus en 1890 après douze années de préparation, dans le $\mathrm{XXI}^{\mathrm{e}}$ chapitre consacré à la perception de la réalité.

${ }^{39}$ Wittgenstein, 1976 [1969], p. 70 ( $\$ 225$; l'auteur souligne). Le fait que nos propositions soient assez fondées à nos yeux, non parce qu'elles ont été éprouvées chacune pour elle-même mais parce qu'elles sont crues ensemble et que les unes fondent les autres, constitue en effet une source d'étonnement philosophique. Voir Wittgenstein, 1976 [1969], p. 61-62: «\$162. [...] J'ai une image du monde. Est-elle vraie ou fausse ? Elle est avant tout le substrat de tout ce que je cherche et affirme. Les propositions qui la décrivent ne sont pas toutes sujettes à vérification. [...] \$166. La difficulté, c'est de nous rendre compte $\mathrm{du}$ manque de fondement de nos croyances. " Pourtant, on peut objecter à Wittgenstein que même si le savoir fonctionne comme une croyance, il n'en demeure pas moins que, s'agissant de la croyance en une divinité qu'évoque aussi le philosophe, les propositions vérifiées qui en accréditent d'autres dans le système font entièrement défaut.

${ }^{40}$ James, 2005 [1897], p. 41, pour les deux citations.
} 
milieu ${ }^{41} \gg$ entrent ici en jeu. Le veto de Clifford intimant au croyant de ne croire que ce qui est prouvé s'avère par conséquent naïf, parce qu'en réalité, en matière de croyance, la libre volonté a peu de place. Du même coup, ce veto confirme la nature passionnelle du rapport aux croyances:

Il [Clifford] croit si fermement à un ordre naturel opposé à la conception chrétienne de l'univers qu'il n'existe pour lui en cette matière aucune option vivante : le christianisme est pour lui dès le début une hypothèse morte ${ }^{42}$.

Le rapport entre la volonté et la croyance n'est d'ailleurs pas à sens unique :

certaines tendances et certaines volitions personnelles précèdent la croyance tandis que d'autres la suivent ; [...] la connaissance et la logique pure, quelle que soit leur puissance idéale, ne constituent pas les seules forces qui, en fait, engendrent nos croyances ${ }^{43}$.

Notre nature passionnelle, décidément, nous conduit toujours à choisir, et même nous fait un devoir d'exercer un choix entre les diverses croyances qui lui sont présentées : puisque « le sentiment de notre devoir vis-à-vis de la vérité et de l'erreur n'est jamais - sachons nous en souvenir - qu'une expression de notre vie passionnelle ${ }^{44} »$, éviter de choisir, comme y invite Clifford, est tout autant une décision passionnelle que de choisir, et revient de fait à choisir.

Pratiquement, la conviction que chacun possède de ne se rendre qu'à l'évidence objective n'est jamais qu'une opinion subjective de plus qui s'ajoute aux autres ${ }^{45}$.

Cela ne signifie aucunement - notons-le passant - que James est un sceptique, et qu'il se refuse à croire que la vérité n'existe pas $^{46}$, mais seulement, en vertu de ce qui vient d'être dit, qu'il estime que le seul moyen d'être fidèle au vœu de vérité est de repousser le dogmatisme spontané et inconscient qui nous tente constamment, pour tâcher d'être " empiristes [...] à la réflexion » - car on ne l'est qu'à travers un effort rigoureux de réflexion ${ }^{47}$. James a cette belle et poétique formule, par où l'on voit qu'il ne limite pas son approche de la croyance aux critères pragmatiques,

\footnotetext{
${ }^{41}$ James, 2005 [1897], p. 45, pour les deux citations. « Dans quatre-vingt-dix-neuf cas sur cent», ajoute James, «notre raison sera satisfaite pourvu qu'elle puisse trouver quelques arguments à réciter machinalement en réponse à une critique éventuelle. Même dans les questions les plus importantes, notre foi n'est le plus souvent que la foi en la foi d'autrui » (James, 2005 [1897], p. 45-46).

42 James, 2005 [1897], p. 49.

43 James, 2005 [1897], p. 47.

44 James, 2005 [1897], p. 53.

45 James, 2005 [1897], p. 51.

${ }^{46}$ Cf. James, 2005 [1897], p. 48, p. 52.

${ }^{47}$ James, 2005 [1897], p. 49.
} 
car la croyance pourrait aussi être une façon d'accueillir d'autres vérités :

L'évidence et la certitude objective constituent certainement un très bel idéal, mais où pourrait-on les rencontrer sur cette planète qu'éclaire la lune et que visite le rêve ? C'est pourquoi je suis moi-même un empiriste complet dans toute l'étendue de ma théorie de la connaissance humaine ${ }^{48}$.

Être empiriste, pour James, c'est être conscient « qu'aucune cloche intérieure ne vient nous signaler la présence de la vérité » de façon indubitable, bien que Clifford, fuyant l'erreur bien plus qu'il ne cherche la vérité, " nous prêch[e] solennellement comme un devoir d'attendre le son de la cloche »: non que nous ne "puissions attendre si cela nous plaît, mais nous le faisons alors à nos risques, exactement comme si croyions. Dans les deux cas nous agissons, nous prenons notre vie entre nos mains ${ }^{49}$. "

On voit à ce qui précède que la volonté entre en compte dans la croyance, pour autant que la nature passionnelle est aussi de nature volitive et émotionnelle. Loin de prétendre que le sujet croit à proprement parler ce qu'il veut croire, comme cela l'enchante, James a montré au contraire que le sujet ne donne son libre assentiment qu'à ce qui lui paraît hypothèse vive, pour des raisons diverses et variées où entrent en ligne de compte des motifs hérités de nos liens sociaux - en tous cas des raisons auxquelles il se rend, parce qu'elles l'ont convaincu, au sens passif de cette expression.

Prenons maintenant le cas où la volonté précède nettement la croyance, c'est-à-dire le cas des croyances auxquelles le sujet veut croire et qu'il se donne pour ainsi dire librement à lui-même. Décider de croire et, ce faisant, créer l'objet de sa foi, la chose est possible, s'agissant de la croyance profane : " combien de cœurs féminins sont domptés simplement par l'ardente insistance de l'homme qui veut en être aimé et qui se refuse à croire que cet amour soit impossible ${ }^{50}$ ! $\gg$ Se faire aimer ou plus simplement plaire suppose, non pas d'attendre dans son coin des éléments de preuve suffisants, mais de témoigner de sa propre affection - ce qui pourrait bien la rendre réciproque. James donne un autre exemple, tiré du Far-West qui s'achève : les bandits attaquant un train sont forts parce qu'ils font équipe; nul voyageur n'ose leur résister, sous peine d'une mort certaine. Mais « si chaque voyageur pouvait seulement croire que tout le wagon réagirait en même temps que lui, il résisterait individuellement, et le pillage serait impossible. Il

\footnotetext{
48 James, 2005 [1897], p. 50.

49 James, 2005 [1897], p. 63.

${ }^{50}$ James, 2005 [1897], p. 58.
} 
$\mathrm{y}$ a donc des cas où un phénomène ne peut se produire s'il n'est précédé d'une foi antérieure en son avènement ${ }^{51}$. »

S'agissant de la croyance religieuse, croire parce que l'on décide de croire sciemment, comme le libertin auquel Pascal propose son célèbre pari, pose cependant problème, puisque c'est un domaine où l'on répugne précisément à croire sous l'effet d'un pari intéressé et quelque peu cynique : il est clair aux yeux de James qu'une croyance acquise de cette façon serait « dépourvue de cette essence intérieure qui fait la réalité de la foi ${ }^{52}$ », de la croyance vivante et sincère. Nous pourrions, avec Bernard Williams, prolonger le propos, et faire remarquer que ce qui pose problème dans ce pari, c'est qu'il faudrait, une fois croyant, que l'on oublie comment on s'est mis à croire si l'on ne veut pas déprécier à ses propres yeux sa croyance ${ }^{53}$.

Toutefois, quel croyant de fraîche date pourrait-il nier l'existence de ce moment où, se rapprochant d'une religion qu'il découvre, redécouvre ou réinterprète s'il s'avère qu'il la connaissait mal, il décide plus ou moins consciemment d'apporter du crédit à ce qui, déjà, a éveillé son intérêt ? Comment faire un départ strict entre le désir de croire et l'attitude consistant à écouter les raisons de croire, qu'elles proviennent d'un témoin, d'une lecture ou de la fréquentation directe des écrits religieux ? La volonté de croire précède la croyance, ou tout du moins lui est concomitante, car pour écouter une parole il faut l'avoir désignée comme digne d'être écoutée - pour des raisons qui peuvent rester à jamais obscures, et dont le croyant pourrait bien ne jamais être pleinement conscient. Bayle le disait déjà avec force :

[...] nous ne croyons les choses que quand elles nous paraissent vraies, et [...] il ne dépend pas de nous qu'elles nous paraissent vraies, non plus qu'il dépend de nous qu'elles nous paraissent blanches ou noires ${ }^{54}$.

Nous n'affirmons comme fondées en raison que les croyances auxquelles nous avons déjà prêté l'oreille parce qu'elles ont déjà commencé à agir en nous - toutes les autres restant lettre morte.

\footnotetext{
${ }^{51}$ James, 2005 [1897], p. 59.

52 James, 2005 [1897], p. 43.

53 Selon Engel, le problème est que le libertin se trouve, au moment de parier sur la foi, en situation de celui qui décide de croire $p$ alors qu'il croit $\neg p$ (cf. Engel, 2007, p. 137).

${ }^{54}$ Bayle, 2006 [1686], p. 150.
} 


\section{CONCLUSION}

Tout comme Clifford a choisi, James choisit - parce que l'esprit humain est ainsi fait - de gager que peut-être "notre croyance obstinée à l'existence des dieux (alors qu'il serait si facile pour la logique comme pour la vie de ne pas croire) rend les plus grands services à l'univers ${ }^{55}$ ". Pour les raisons qu' on a dites avec Kant, " personne ne peut lui démontrer irréfutablement ${ }^{56}$ » qu'il se trompe. Le plus énigmatique, pour James, tient à ce que, «tout en étant, en un sens, des parties passives de l'univers, nous [fassions] preuve, d'un autre côté, d'une curieuse autonomie, comme si nous étions pour notre propre compte de petits centres d'action ${ }^{57} \gg$, comme si l'univers comptait sur notre décision - quelle qu' elle soit.

On croit parce qu' on veut croire ; on ne croit pas parce qu'on ne veut pas croire. L'athéisme, en tant que croyance en l'inexistence de réalités suprasensibles, constitue une forme de croyance : selon James, il est seulement une «complication» de la croyance ${ }^{58}$. L'agnosticisme, à strictement parler, n'en constitue pas une, mais, à lire là encore James, on comprend qu'il est rare, ou plutôt qu'il se contredit fatalement par le critère de l'action : même sans se prononcer théoriquement sur le caractère existant ou non de réalités suprasensibles, il n'en statue pas moins sur le caractère inexistant, ou fortement improbable, de ces mêmes réalités, puisque vivre concrètement en ne comptant que sur les seules forces (immanentes) de l'humain, c'est agir comme si rien de divin dans l'Univers n'existait.

Dans notre volonté de croire ou de ne pas croire se manifestent des raisons, qui sont pleinement des raisons, mais dont on serait bien en peine de dire pourquoi elles emportent notre adhésion alors que d'autres, inverses, le pourraient aussi. Notre passion d'affirmer et de nier se mêle à nos raisons, de sorte que seront raisonnables à nos yeux... les motifs d'affirmer qui auront le bonheur de nous plaire. La dimension émotionnelle de nos croyances se voit bien au fait que celles-ci, comme toute chose dans nos vies humaines, ont leur mouvement de crue et de décrue ${ }^{59}$. Elle se manifeste mieux encore au fait que, quel que soit le degré de vivacité avec lequel

\footnotetext{
55 James, 2005 [1897], p. 62.

${ }^{56}$ James, 2005 [1897], p. 64.

${ }^{57}$ James, 2005 [1897], p. 61.

${ }^{58}$ James, 2010, [1889], p. 102.

${ }^{59}$ Dans « La psychologie de la croyance » (James, 2010 [1889], p. 136) James cite un propos d'Emerson qui n'est pas sans rapport avec le nôtre : « Notre foi vient par moments [...] mais il y a une intensité dans ces brefs moments qui nous contraint à leur attribuer plus de réalité qu'aux autres expériences ».
} 
nous affirmons ou nions des croyances, c'est toujours leur état présent qui nous semble le plus juste. Montaigne l'avait compris :

Que la fortune nous remue cinq cents fois de place, qu'elle ne fasse que vider et remplir sans cesse, comme dans un vaisseau [vase], dans notre croyance autres et autres opinions, toujours la présente et la dernière c'est la certaine et l'infaillible ${ }^{60}$.

C'est une leçon de modestie, en ce sens, que l'on peut tirer de cet examen des raisons de la croyance en lien avec la volonté de croire, tant est vaste l'espace de variations du croire, tant le croyable de l'un est l'incroyable de l'autre, tant chacun varie dans la nature et le degré de ses affirmations, car si les institutions et les écritures sacrées régulent le champ religieux, elles génèrent ellesmêmes d'importantes variations dans la façon dont elles sont comprises, et suivies - pour ne rien dire de la liberté immense par laquelle chaque croyant, en son âme de conscience, se place seul devant le divin qu'il invoque. Le croyant croit certes "parce qu'il est écrit...", mais dans l'assentiment qu'il donne aux écritures sacrées qui fondent sa foi, il les conforte dans leur rôle d'autorité, si bien que la relation entre l'autorité et celui qui la reconnaît est circulaire, ou en spirale, l'une renforçant l'autre ${ }^{61}$. $\mathrm{Si}$, aux yeux du croyant et religieusement parlant, c'est le message ou la pratique qui justifie tout, c'est, philosophiquement parlant, le sujet croyant qui choisit de se fier à cette autorité, de sorte que la responsabilité de ses croyances lui incombe, d'abord sur le plan épistémique même si, redisons-le, il est ardu de savoir pourquoi nous sommes convaincus par nos raisons - et surtout sur le plan moral.

Car il est sûr qu'il n'y aura pas de scepticisme là où entrent en jeu les implications morales de la croyance. Face au caractère parfois presque vain de la justification des croyances, on tendra à désigner comme signe distinctif de la croyance ce qu'elle engage le croyant à faire ou à ne pas faire: "une question religieuse est seulement ou bien une question de vie [Lebensfrage] ou bien un bavardage (vide) ${ }^{62} »$. Il a fallu des siècles pour que l'appel à la violence religieuse paraisse pour ce qu'il est, un crime odieux contre l'humain, d'autant plus odieux qu'il est perpétré au nom de la religion. Il a fallu des siècles pour qu'enfin l'hérétique et l'athée

\footnotetext{
${ }^{60}$ Montaigne, cité par Brahami, 1997, p. 72.

${ }^{61}$ Il est étrange qu'au sein des traditions religieuses, l'on ne soit pas davantage conscient $\mathrm{du}$ fait que les raisons de croire, qu'elles proviennent de ma propre expérience ou $\mathrm{du}$ témoignage d'autrui, supposent nécessairement que moi, comme sujet, je les valide.

${ }^{62}$ Wittgenstein (dans ses Carnets de Cambridge et de Skjolden), cité par Bouveresse, 2007, p. 238-239. James notait aussi : "Le critérium expérimental de la valeur des hypothèses religieuses relatives à l'univers, le seul moyen qui nous soit offert de les déclarer vraies ou fausses, réside dans l'examen des croyances individuelles actives et de leur expression spontanée dans la vie » (James, 2005 [1897], p. 36).
} 
ne soient plus considérés comme des criminels sous nos latitudes ; on le doit à des esprits comme Pierre Bayle, dont le nom doit une fois de plus être salué, soucieux (dans ses Pensées diverses sur la comète $^{63}$ comme dans son traité De la tolérance ${ }^{64}$ ) de chercher à comprendre l'incroyance, de condamner les croyances superstitieuses et l'idolâtrie. De tels penseurs, eux-mêmes croyants, ont permis de comprendre que l'hérétique et l'athée ne sont pas moins que les croyants sincèrement liés, en leur âme et conscience, par des raisons de croire différemment, ou de ne pas croire.

Mais la ligne de partage entre les croyances recevables et celles qui ne le sont pas n'a rien à voir avec la sincérité de la croyance ; elle est, répétons-le, d'ordre moral. Dire que chacun croit ce qu'il peut, selon ce qui est en son pouvoir, ne signifie pas que chacun n'a de compte à rendre qu'à soi-même et à son Dieu. Car chacun est comptable de ses croyances pour autant qu'elles engagent un comportement qui, lui, peut et doit faire l'objet d'une évaluation commune. L'examen de ce qui produit la croyance cède ici le pas à ce que produit la croyance sur l'individu et la société ; examen dont on sait que les philosophes n'ont pas eu trop de mal à l'apporter au discrédit des croyants. Avec l'ensemble des philosophes, disons qu'il n'y a pas de violence sacrée : il n'y a que de la violence. Les religions ont été les témoins de la violence enracinée en l'homme, en ceci que les écritures sacrées et les témoignages de l'histoire ont montré les hommes comme ils sont, enclins à la violence, au monopole de la vérité ; elles ont été aussi - ce serait hypocrite de le taire - des facteurs de violence, autant que de paix.

Une réflexion distanciée sur la façon dont se construit la croyance, sur les mécanismes de persuasion intérieure, peut avoir un effet cathartique. Elle peut faire accepter que, comme toute chose humaine, l'adhésion à une conviction épouse la volonté d'avoir raison, d'être soi dans le vrai - ce qui est normal, étant donné que la croyance est un «tenir pour vrai »- mais aussi que cette adhésion peut être le signe d'une volonté de l'emporter, de s'imposer, comme si c'était l'objet de la conviction (Dieu, tel Dieu, telle religion) qui l'exigeait et non pas le croyant lui-même. Que ce dernier, s'il professe aujourd'hui une conviction forte qui semble maintenant la sienne au plus profond de son être, se demande s'il était pas aussi lui-même lorsqu'il ne l'avait pas - car on ne naît pas avec des convictions, on les acquiert. Il pourrait bien alors, non pas renoncer à sa conviction, puisque nous sommes des êtres de conviction, mais comprendre la part que lui, humainement, y prend ; il pourrait ainsi

\footnotetext{
${ }^{63}$ Bayle, 2007 [1682].

${ }^{64}$ Voir Bayle, 2006 [1686] ; cf. Frey, 2014.
} 
se demander quel est le meilleur hommage qu'il puisse rendre à ces idées plus hautes et plus anciennes que lui. Car si la croyance peut être, hélas, infidèle aux exigences éthiques minimales, elle peut aussi posséder un réel pouvoir heuristique : le pouvoir d'inciter au bien, sous toutes ses formes, et de révéler des ressources de vie et de bonté qui, sans la croyance, auraient bien pu demeurer insoupçonnées.

\section{BIBLIOGRAPHIE}

Anselme, 1947 [XI $\mathrm{XI}^{\mathrm{e}}$ siècle]: Anselme de Cantorbéry, Proslogion $\left[\mathrm{XI}^{\mathrm{e}}\right.$ siècle], in : Euvres philosophiques de Saint Anselme: Monologion, Proslogion, De veritate, De libero arbitrio, De concordia, De voluntate. Traduction de P. Rousseau, Paris, Aubier, 1947, p. 175-221.

Bayle, 2006 [1686], Pierre Bayle, De la tolérance. Commentaire philosophique, J.-M. Gros (éd.), Genève, Honoré Champion, 2006 (Champion classiques littérature).

Bayle, 2007 [1682], Pierre Bayle, Pensées diverses sur la comète, J. et H. Bost (éd.), Paris, Flammarion, 2007.

Bouveresse, 2007: Jacques Bouveresse, Peut-on ne pas croire? Sur la vérité, la croyance \& la foi, Marseille, Agone, 2007 (Banc d'essais).

Bouveresse, 2015 : Jacques Bouveresse, L'éthique de la croyance et la question du "poids de l'autorité ", Paris, Collège de France, 2015 (édition numérique disponible via books.openedition.org).

Brahami, 1997 : Frédéric Brahami, Le scepticisme de Montaigne, Paris, PUF, 1997 (Philosophies).

Clifford, 1879 [1877] : William Kingdon Clifford, «The Ethics of Belief » [1877], in: Lectures and Essays, vol. II., L. Stephen and F. Pollock (éd.), Londres, Mac Millan, 1879, p. 177-211.

Engel, 1995 : Pascal Engel, «Les croyances », in : Notions de philosophie II. Sous la direction de D. Kambouchner, Paris, Gallimard, 1995, p. 9-100.

Engel, 2007 : Pascal Engel, Va savoir! De la connaissance en général, Paris, Hermann Éditeurs, 2007.

Frey, 2014 : Daniel Frey, «Faux et usage de faux. La dénonciation du mésusage de Lc 14,23 dans le Commentaire philosophique de Pierre Bayle ", in: Usages et mésusages de l'Écriture. Approches interdisciplinaires de la référence scripturaire. Textes réunis par D. Frey, C. Grappe et M. Wieger, Strasbourg, PUS, 2014, p. 177-192 (Écriture et Société).

Frey 2015 : Daniel Frey, « La croyance de l'enfant selon Rousseau. Émile à la lumière des philosophie et psychologie de la religion », RHPR 95, 2015, p. 265-287. 
Gaunilon, 1947 [XI ${ }^{\mathrm{e}}$ siècle] : Gaunilon, «Pour la défense de l'insensé » [XI ${ }^{\mathrm{e}}$ siècle], in : Euvres philosophiques de Saint Anselme: Monologion, Proslogion, De veritate, De libero arbitrio, De concordia, De voluntate. Traduction de P. Rousseau, Paris, Aubier, 1947, p. 201-207 (Bibliothèque philosophique).

James, 2005 [1897] : William James, La volonté de croire [1897]. Traduction de L. Moulin (Flammarion, 1916), Paris, Le Seuil - Les Empêcheurs de penser en rond, 2005.

James, 2010 [1889] : William James, «Psychologie de la croyance » [1889], in : La psychologie de la croyance, et autres essais pragmatistes. Traduction S. Galetic, Nantes, Editions Cécile Defaut, 2010.

Kant, 1968 [1790] : Emmanuel Kant, Critique de la faculté de juger [1790]. Traduction d'A. Philonenko, Paris, Vrin, 1968 (Bibliothèque des textes philosophiques).

Kant, 1980 [1781/1787] : Emmanuel Kant, Critique de la raison pure [1781/1787], in : Id., Euvres philosophiques. I, Des premiers écrits à la «Critique de la raison pure ». Édition publiée sous la direction de F. Alquié, Paris, Gallimard, 1980, p. 705-1470 (Bibliothèque de la Pléiade).

Meulders, 2010 : Michel Meulders, William James. Penseur libre, Paris, Hermann Éditeurs, 2010

Pascal, 1963 [1662] : Blaise Pascal, Pensées [1662], in : Id., CEuvres complètes. Présentation et notes de L. Lafuma, Paris, Seuil, 1963, p. 493-647.

Platon, 1967 : Platon, Théétète, in : Théétète. Parménide. Traduction d'É. Chambry, Paris, Garnier-Flammarion, 1967.

Pouivet, 2002 : Roger Pouivet, "Épistémologie de la croyance religieuse », in: S. Bourgeois-Gironde, B. Gnassounou et R. Pouivet (éd.), Analyse et théologie. Croyances religieuses et rationalité, Paris, Vrin, 2002, p. 11-30 (Problèmes \& controverses).

Pouivet, 2003 : Roger Pouivet, Qu'est-ce que croire?, Paris, Vrin, 2003 (Chemins philosophiques).

Pouivet, 2013 : Roger Pouivet, Épistémologie des croyances religieuses, Paris, Cerf, 2013 (Philosophie \& théologie).

Rey, 1998 : Alain Rey, « Croire », in : Dictionnaire historique de la langue française dirigé par Alain Rey, Paris, Dictionnaires Le Robert, 1998, p. 956-957.

Ricœur, 2000 [1969] : Paul Ricœur, «Croyance» [1969], in : Dictionnaire de la philosophie, Paris, Encyclopædia Universalis - Albin Michel, 2000, p. 309-328.

Rousseau, 1969 [1762] : Jean-Jacques Rousseau, Émile, ou de l'éducation [1762], in : Id., Euvres complètes, IV, Émile. Éducation - Morale - Botanique. Édition publiée sous la direction de B. Gagnebin et M. Raymond, Paris, Gallimard, 1969, p. 239-868 (Bibliothèque de la Pléiade).

Williams, 2006 [2002] : Bernard Williams, Vérité et véracité. Essai de généalogie [2002]. Traduction de J. Lalaidier, Paris, Gallimard, 2006 (NRF essais).

Wittgenstein, 1976 [1969]: De la certitude [1969]. Traduction de J. Fauve, Paris, Gallimard, 1976 (Tel). 